INVESTIGACIÓN

\title{
EVALUACIÓN DE LA CALIDAD DEL AGUA DEL RÍO TEJO (OCAÑA, COLOMBIA) MEDIANTE MACROINVERTEBRADOS ACUÁTICOS Y PARÁMETROS FISICOQUÍMICOS
}

\section{ASSESSMENT OF WATER QUALITY OF RIVER VALLEY (OCAÑA, COLOMBIA) MACROINVERTEBRATES BY WATER AND PHYSICOCHEMICAL PARAMETERS}

\author{
Msc. José Julián Cadena Mª ; Esp. Rubén Darío Gómez T ${ }^{\mathrm{b}}$ \\ ${ }^{\text {a } U n i v e r s i d a d ~ F r a n c i s c o ~ d e ~ P a u l a ~ S a n t a n d e r ~ O c a n ̃ a, ~ G r u p o ~ d e ~ I n v e s t i g a c i o ́ n ~ G I F E A H, ~ V i ́ a ~ A c o l s u r e, ~}$ \\ Sede Algodonal, Ocaña, Colombia, ijcadenam@ufpso.edu.co \\ ${ }^{\mathrm{b}}$ Universidad Francisco de Paula Santander Ocaña-Grupo de Investigación GIFEAH- Vía Acolsure, \\ Sede Algodonal, Ocaña, Colombia, rdgomezt@ufpso.edu.co
}

Fecha de recepción: 29-02-2016

Fecha de aprobación: 05-05-2016

Resumen: Se evalúo la calidad del agua del Río Tejo en tres tramos de monitoreo durante los meses de mayo, junio y julio de 2014, mediante el uso de macroinvertebrados acuáticos y parámetros fisicoquímicos, valorados posteriormente con el uso de los índices Biological Monitoring Working Party (BMWP), ICA-NSF e índices de diversidad para cada unidad de estudio. Los índices ecológicos de diversidad, riqueza y equidad fueron relativamente bajos, confirmando aguas moderadamente contaminadas. Según el BMWP, arrojó aguas aceptables, ligeramente contaminadas o se evidencian efectos de contaminación, lo cual fue confirmado al realizar el comparativo con el ICA-NSF en donde se evidencia una calidad mediana en cada una de los tramos evaluados.

Palabras clave: Índice Calidad de aguas, Parámetros físicoquímicos, Índices de Diversidad, Valoración de la calidad de ríos.

Abstract: Water quality of the River Tejo in three installments of monitoring was evaluated during the months of May, June and July 2012, using aquatic macroinvertebrates and physicochemical parameters, then valued using indices Biological Monitoring Working Party (BMWP), ICA-NSF and diversity indices for each unit of study.The ecological 
diversity indices, richness and evenness were relatively low, confirming moderately polluted waters. According BMWP, courage acceptable water slightly contaminated or contamination effects are evident, which was confirmed by performing the comparison with the ICA-NSF where medium qualities in each of the sections are evaluated evidence.

Keywords: Water quality index, physicochemical parameters, diversity indices, rating the quality of rivers.

\section{INTRODUCCIÓN}

El uso de macroinvertebrados para valorar y determinar la calidad del agua, tiene cuando menos 100 años de antigüedad. De estas técnicas, los insectos acuáticos (entre un $70-90 \%$ de la fauna de macroinvertebrados dulceacuícolas) han sido el grupo más estudiado para evaluar la calidad del agua por muchos investigadores (Hellawell, 1986; Abel, 1989; Rosenberg \& Resh, 1993, entre otros). De esta manera, la heterogeneidad física y química, incluyendo el sustrato y la velocidad de la corriente en el canal del rio, constituyen un factor importante que puede influenciar la diversidad biótica de éste (Roldan, 1996). Por tal razón, la composición de la estructura de estas comunidades ha adquirido una creciente importancia en los estudios de los ecosistemas acuáticos debido a que las variables fisicoquímicas sólo dan una idea puntual sobre la calidad del agua y no informan sobre las variaciones en el tiempo (Alba-Tercedor, 1996).

En este sentido, los métodos biológicos para la determinación de la calidad de agua han sido utilizados en Europa desde principios de siglo; sin embargo en la década de los cincuenta se tuvo mayor consideración en la respuesta que ofrecían las plantas y animales como evidencia directa de la contaminación. Estos métodos, básicamente trabajan sobre la premisa de la tolerancia o nivel de respuesta que los macroinvertebrados presenten frente a determinados factores. De esta manera, se resalta los métodos propuestos por Wilhm \& Dorris (1968), al igual que Staub et al, (1970), en el que aplican los índices de diversidad de Shannon-Weaver para determinar la contaminación por materia orgánica, los cuales son útiles y de fácil aplicación. Para nuestro caso, además de utilizar índices de dominancia, diversidad y equidad, se hizo indispensable la utilización de los índices BMWP Colombia (Roldan, 2003), que permite en un corto tiempo determinar las puntuaciones ecológicas de las familias según su grado de tolerancia a la eutrofización, sumados al Índice ICA-NSF que en la actualidad es utilizado para supervisar la calidad de los ríos a través del tiempo y comparar aguas de abastecimiento en muchos países del Mundo (NSF, 2006).

Así mismo, partiendo de los escasos estudios acerca de la calidad del agua del 
río Tejo e importancia que representa dicho recurso, fue indispensable contar con herramientas concretas de gestión del recurso hídrico. Es por ello, que el presente estudio sobre diversidad de macroinvertebrados acuáticos, realizado bajo la metodología de investigación empírica de evaluación de la calidad del agua del rio Tejo, busca, mediante índices biológicos y fisicoquímicos valorar las aguas de la cuenca en un tramo de $1.5 \mathrm{~km}$ subdividido en tres estaciones de monitoreo, comprendiendo 300 metros antes de entrar a la zona urbana del municipio de Ocaña y 100 metros antes de la confluencia con la Quebrada Juan Sánchez.

\section{MATERIALES Y MÉTODOS}

\section{Área de Estudio}

El estudio se realizó en la Cuenca del Rio Tejo, en la zona de influencia de la Vereda la Pradera, que cuenta con 155.4 ha., localizada en el municipio de Ocaña (Norte de Santander, Colombia). Está situada a $8^{\circ} 14^{\prime} 15^{\prime \prime} \mathrm{N}$ y $73^{\circ} 2^{\prime} 26^{\prime \prime} \mathrm{W}$ y su altura sobre el nivel del mar corresponde $1.202 \mathrm{~m}$. La localidad donde se ubican los puntos de monitoreo posee una temperatura media de $22^{\circ} \mathrm{C}$ y precipitaciones promedio anuales de 1500 mm. La zona de vida según Holdridge (1996), corresponde a Bosque Seco premontano (Bs-Pm).

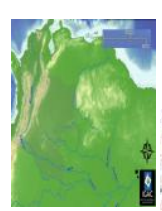

Figura 1. Sitio de estudio en el rio Tejo, Ocaña, Norte de Santander.

Fuente: Tomada de Instituto Geográfico Agustín Codazzi

El río Tejo, que durante varios siglos fue la principal fuente de agua potable de los

ocañeros, tiene una extensión $20 \mathrm{Km}$, nace en La Reserva Forestal Cuchilla de Simitarigua, Vereda Sabaneta, municipio de Ocaña, y confluyen sus aguas en límites con el municipio de San Calixto (Norte de Santander), donde entrega sus aguas al Rio Catatumbo.

\section{Selección del área, duración del tiempo de muestreo y recolección de muestras}

La recolección de la información se llevó a cabo en mayo, junio y julio del año 2014, los cuales corresponden a las unidades $\boldsymbol{M 1}$, M2 y M3. Los sitios de muestreo fueron escogidos teniendo en cuenta los cultivos de fincas aledañas, los asentamientos humanos y por el fácil acceso en el momento de realizar el monitoreo, buscando la cercanía al municipio de Ocaña en un tramo aproximado de $1.5 \mathrm{~km}$. Las unidades se ubican $300 \mathrm{~m}$ antes de entrar a la zona urbana del municipio de Ocaña y 100 metros antes de la confluencia con la Quebrada Juan Sánchez (Figura 1). El periodo de monitoreo no coincidió altamente con precipitaciones fuertes siendo muy notoria la época de estiajes. 
Los tramos para la recolección de datos estuvieron separados aproximadamente $450 \mathrm{~m}$ el uno del otro, tomándose un área mínima de monitoreo de 30 metros en cada punto, teniendo como longitud total una extensión de $1.5 \mathrm{~km}$.

La recolección de material, en cada sitio de muestreo se realizó a través de tres réplicas multihábitat de los macroinvertebrados bentónicos, utilizando una red circular (0.02 $\mathrm{m}^{2}, 250 \mu \mathrm{m}$ de poro), disturbando los microhábitats en una sección transversal del rio de un lado al otro de la ribera durante 30 minutos. Los individuos encontrados se extrajeron in situ y fueron fijados en alcohol $80 \%$ para su posterior identificación en el laboratorio. Cada individuo se identificó en las categorías Orden, Familia y en lo posible la especie en el que se incluían la totalidad de individuos por cada taxa. Esta práctica se realizó cada mes, siendo escogidos 15 días consecutivos en cada punto de monitoreo.

El análisis fisicoquímico y de coliformes fecales se realizó en el laboratorio de Aguas de la Universidad Francisco de Paula Santander de Ocaña, mediante muestras compuestas, en los mismos puntos donde se realizaron los muestreos biológicos y al mismo tiempo que estos.

\section{Tratamiento de los Datos}

Para describir la estructura numérica de la comunidad de macroinvertebrados se utilizaron los índices de:

Diversidad de Shannon - Weaver (H'). Este índice se mide en unidades de bits/individuo cuando la escala logarítmica es base 2. En el contexto de los ecosistemas fluviales este índice adquiere un valor máximo de 4,5 bits/individuo para las comunidades de macroinvertebrados bentónicos. Valores inferiores a 2,4 - 2,5 bits/individuo son indicadores de que el ecosistema se encuentra sometido a tensión (vertidos, dragados, canalizaciones, regulación por embalses, etc.). Este índice disminuye mucho en aguas muy contaminadas.

Dominancia de Simpson (D). Este índice muestra la abundancia de las especies más comunes, por lo tanto se refiere al mayor número de especies representadas en la muestra total de individuos, su valor va de 0 a 1.

Riqueza de Margalef (R). Se refiere al mayor número de individuos representados en cada una de las muestras.

Equidad de Pielou (E). Se refiere a la distribución de los taxones en las diferentes estaciones de muestreo, su valor va de 0 a 1.

Similitud de Jaccard (S). Este índice calcula la semejanza entre las comunidades de macro invertebrados encontrados en cada una de las unidades de monitoreo.

BMWP Col (Biological Monitoring Working Party). Índice biológico que se computa sumando las ponderaciones asignadas a los distintos taxones encontrados en las muestras de macroinvertebrados y que se citan en una lista elaborada al respecto. La mayor o menor puntuación asignada a un taxón está en función de su mayor o menor sensibilidad a la contaminación orgánica y al déficit de oxigeno que este tipo de contaminación suele provocar en la mayor parte de los ríos y quebradas (Roldán, 2003).

\section{ICA-NSF}


Propuesto en 1970, este índice es ampliamente utilizado para medir la calidad del agua y puede ser utilizado para medir los cambios en tramos particulares de los ríos a través del tiempo comparando la calidad del mismo. En la

\section{RESULTADOS Y DISCUSIÓN}

En el rio Tejo se recogieron 85 macroinvertebrados, distribuidos en 6 órdenes y 13 familias (Tabla 1), siendo la (1. 17\%), La familia más representativa la Coenagrionidae con 21 individuos (24.70\%), seguida de Naucoridae con 20 individuos (23.52\%), Beatidae y Leptophlebiidae con 9 individuos (10.58\%), Hidrobiosidae con 6 individuos (7.05\%), Gomphidae con 5 individuos $(5.88 \%)$, Tipulidae con 4 individuos

(4.70\%), Libellulidae y Velidae con 3 individuos cada uno (3.52\%) Oliygoneuridae 2 individuos (2.35\%).Contrastando con lo anterior, las familias que presentaron la menor abundancia fueron Tubificidae, Psychodidae, Aeshnidae, cada una con un individuo actualidad es utilizado para supervisar la calidad de los ríos a través del tiempo y comparar aguas de abastecimiento en Estados Unidos y muchos países del Mundo (NSF, 2006).

Propuesto en 1970, este índice es ampliam a nivel de órdenes, el que presentó mayor representatividad fue Odonata con 4 familias $(30.76 \%)$, seguida de Ephemeroptera con 3 familias (23.07\%), sucedido por Hemíptera y Díptera con 2 familias cada uno $(15.38 \%)$. En este sentido, los órdenes Trichoptera y Haplotaxida se registraron con una familia cada uno (7.69\%). Hay que destacar que el orden Odonata se registró con 4 familias (30.76\%) y a nivel de abundancia de individuos su aporte es el de mayor número del total de la muestra (35.29\%). Ephemeroptera se registró con 3 familias $(23.07 \%)$, por lo que su abundancia de individuos fue menor que Odonata (23.52\%) y a diferencia del orden Hemíptera que a pesar de estar representados por 2 familias $(15.38 \%)$ su número de individuos es mayor con $27.05 \%$.

En el muestreo uno (M1), se encontraron 19 individuos $(22.35 \%)$, pertenecientes a 5 familias y 2 ordenes, siendo la familia más abundante Naucoridae con 8 individuos $(42.1 \%$ del total de encontrados en este punto de muestreo; seguida de Libelludidae, Gamphidae, Velidae, con 3 individuos cada uno $(15.78 \%)$, la familia menos representativa fue Coenagrindae con 2 individuos $(10.52 \%) \mathrm{y}$, el orden mejor representado en esta unidad fue Odonata con 3 familias (60\%), seguida de Hemíptera con 2 familias (40\%) (Tabla 2).

En el muestreo dos (M2), se registraron 31 individuos $(36.47 \%)$, distribuidos en 6 familias y 4 órdenes. $\mathrm{La}$ familia predominante fue Coenagrindae con 11 individuos $(35.48 \%)$, seguida por Naucoridae con 6 individuos (19.35\%), Leptophlebiidae con 5 individuos (16.12\%), Beatidae 4 individuos (12.9\%), Tipulidae 3 individuos $(9.67 \%)$, y la menos representativa corresponde a Gomphidae con 2 individuos $(6.45 \%)$. En este muestreo los órdenes más abundantes son Odonata y Ephemeroptera con 2 familias cada uno, (33.33\%) seguida Hemíptera, y Díptera con 1 familia cada una $(16.66 \%)$ (Tabla 3) 
Tabla1. Composición taxonómica de la comunidad de macroinvertebrados recolectados en el rio Tejo, sitio La Pradera, municipio de Ocaña Norte de Santander, Colombia

\begin{tabular}{llc}
\hline & ORDEN & NAMILIA DE INDIV \\
\hline Odonata & Coenagrionidae & 21 \\
& Gomphidae & 5 \\
& Libellulidae & 3 \\
Ephemeroptera & Aeshnidae & 1 \\
& Leptophlebiidae & 9 \\
Hemíptera & Oliygoneuridae & 2 \\
& Naucoridae & 20 \\
Díptera & Velidae & 3 \\
Hrichoptera & Tipulidae & 4 \\
\hline Fuplotaxida & Psychodidae & 1 \\
\hline
\end{tabular}

Fuente: elaboración propia.

Tabla 2. Individuos encontrados en el muestreo 1(M1) en el rio Tejo sitio La Pradera municipio de Ocaña Norte de Santander, Colombia.

\begin{tabular}{ccc}
\hline ORDEN & FAMILIA & No DE INDIVID \\
\hline \multirow{2}{*}{ Odonata } & Coenagrionidae & 2 \\
& Libelludidae & 3 \\
\multirow{2}{*}{ Hemíptera } & & 3 \\
& Gomphidae & 8 \\
\hline
\end{tabular}

Fuente: Elaboración propia

Tabla 3. Individuos encontrados en el muestreo 2(M2) del rio Tejo sitio La Pradera, Municipio de Ocaña, Norte de Santander, Colombia.

\begin{tabular}{ccc}
\hline ORDEN & FAMILIA & NUMERO DE INDIVIDUOS \\
\hline Odonata & Coenagrionidae & 11 \\
& Gomphidae & 2 \\
Ephemeroptera & Beatididae & 4 \\
\hline
\end{tabular}




\begin{tabular}{ccc}
\hline & Leptophlebiidae & 5 \\
Hemíptera & Naucoridae & 6 \\
Díptera & Tipulidae & 3 \\
\hline
\end{tabular}

Fuente: Elaboración propia

En el muestreo tres (M3), se hallaron 35 individuos $(41.17 \%)$, pertenecientes a 10 familias y 6 órdenes. La familia que presentó mayor cantidad de individuos fue Coenagrionidae con 8 individuos $(22.85 \%)$, seguida de Naucoridae, Hidrobiosidae con 6 individuos cada una (17.14\%), Beatidae con 5 individuos (14.28\%), Leptophlebiidae con 4 individuos (11.42\%), Oligoneuriidae con 2 individuos (5.71\%), y las familias de menor representación fueron Tubificidae, Tipulidae, Psychodidae y Aeshnidae con 1 individuo cada una $(2.85 \%)$. De acuerdo al orden, el de mayor representación fue Ephemeroptera con 3 familias (30\%), seguida de Diptera y Odonata con 2 familias cada una (20\%), Hemíptera, Haplotaxida y Trichoptera con una familia cada una (10\%). Cabe destacar que en este muestreo fue el único donde se encontró las familias Hidrobiosidae (Trichoptera) y $\quad$ Tubificidae (Haplotaxida).

Los cambios en la estructura numérica de la comunidad de macroinvertebrados fueron relativamente pocos. La mayor diversidad de macroinvertebrados acuáticos se registró en el muestreo 3 (2.03), lo cual se asume que se debe a las variaciones climáticas ya que el rio presentó de leve a suave aumento de caudal por lo que pudo ocurrir un arrastre de macroinvertebrados de las Unidades M1 y M2 o zonas intermedias de estos.

Tabla 4. Individuos encontrados en el muestreo 3 (M3) del rio Tejo, sitio La Pradera municipio de Ocaña, Norte de Santander. Colombia

\begin{tabular}{ccc}
\hline ORDEN & FAMILIA & NUMERO DE INDIV. \\
\hline \multirow{2}{*}{ Ephemeroptera } & Leptophlebiidae & 4 \\
& Beatidae & 5 \\
Díptera & Oligoneuriidae & 2 \\
& Tipulidae & 1 \\
Odonata & Psychodidae & 8 \\
& Coenagrionidae & 1 \\
Haplotaxida & Aeshnidae & 6 \\
& Naucoridae & 1 \\
Trichoptera & Tubificidae & 6 \\
\hline
\end{tabular}

Fuente: Elaboración propia

Por otra parte, podemos afirmar que estos cambios en cada muestreo se deben también a las perturbaciones ocasionadas por vertidos domésticos y agrícolas difusos y puntuales al rio, sumados a la 
extracción de arena, intermedio a la Unidad M1-M2. Sin embargo, cuando comparamos las tres unidades se presenta poca diferencia, la riqueza varía de 1.46 a 2.53, presentando en el muestreo 3 el mayor valor. La dominancia fue baja en los 3 muestreos presentando su máximo valor en el muestreo 1 (0.19), lo que explica que en las tres unidades la dominancia está dada por aquellas familias con mayor número de individuos. Este comportamiento está asociado con una mayor abundancia de la familia Coenagrionidae con 21 individuos (24.70\%), mientras la equidad resultó ser alta en los tres muestreos.

Tabla 5. Valores de los índices ecológicos obtenidos para cada muestreo en el rio Tejo, sitio La Pradera, municipio de Ocaña.

\begin{tabular}{cccc}
\hline ÍNDICES & M1 & M2 & M3 \\
\hline Riqueza & 2.17 & 1.46 & 2.53 \\
Diversidad & 1.51 & 1.61 & 2.03 \\
Equidad & 0.77 & 0.84 & 0.88 \\
\hline Dominancia & 0.19 & 0.17 & 0.14 \\
\hline
\end{tabular}

Fuente: Elaboración propia

Según la puntuación del BMWP (Biological Monitoring Working Party), este sistema puede considerarse como Aguas con Calidad Aceptables (Categoría II: 61-100), ligeramente contaminadas, que evidencian efectos de contaminación con un puntaje de 78 , siendo catalogado como un sistema oligotrófico (Tabla 6).
Esto permite visualizar, que aparentemente el rio tiene una calidad adecuada antes de entrar a la zona urbana de Ocaña, en donde las condiciones son significativamente muy malas, debido a los vertidos puntuales aguas residuales domésticas, comerciales, institucionales e industriales que sobre él se descargan.

Tabla 6. Puntajes asignados a las familias de macroinvertebrados según el BMWP, según Roldan (2003).

\begin{tabular}{|c|c|c|}
\hline FAMILIA & PUNTAJE BMWP & INDIVIDUOS \\
\hline Coenagrionidae & 7 & 21 \\
\hline Naucoridae & 8 & 20 \\
\hline Gomphidae & 9 & 5 \\
\hline Tipulidae & 3 & 4 \\
\hline Beatidae & 7 & 9 \\
\hline Hidrobiosodae & 5 & 6 \\
\hline Leptophlebiidae & 9 & 9 \\
\hline Libellulidae & 5 & 3 \\
\hline Velidae & 7 & 3 \\
\hline Oliygoneuridae & 9 & 2 \\
\hline Tubificidae & 1 & 1 \\
\hline Psychodidae & 2 & 1 \\
\hline
\end{tabular}




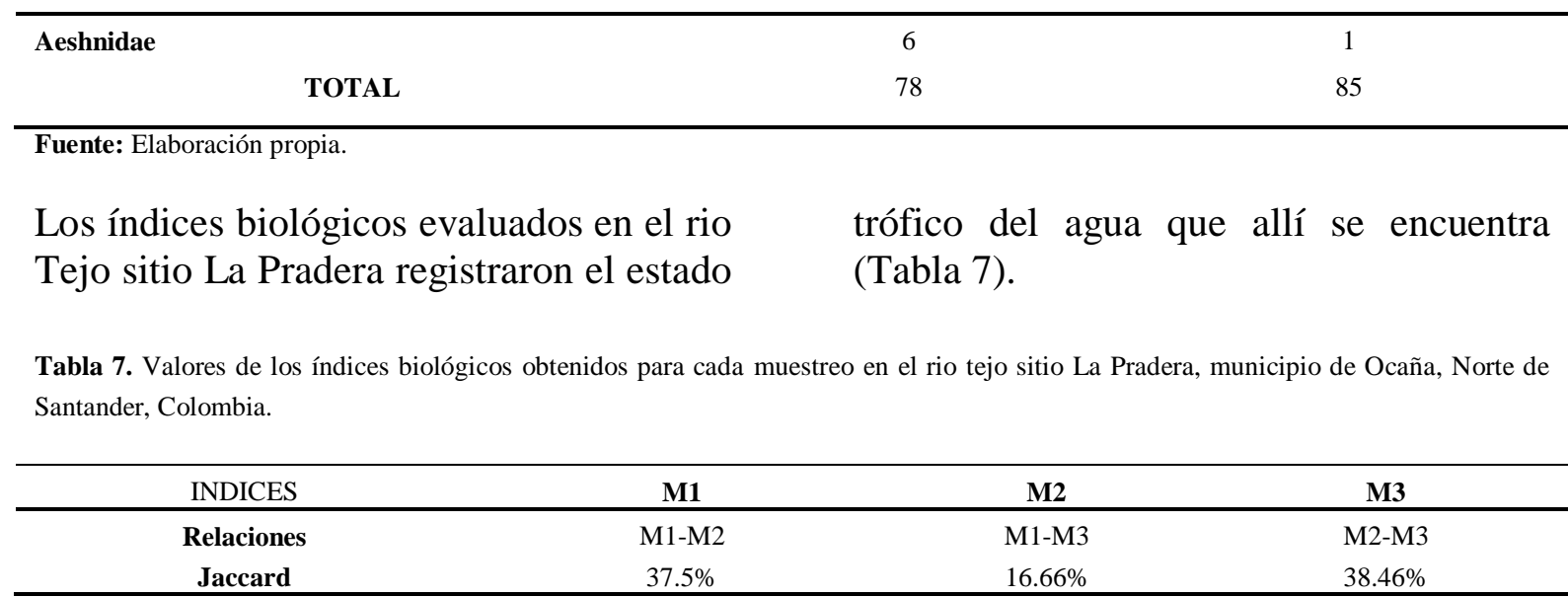

Fuente: Elaboración propia

Por otra parte, siguiendo los lineamientos de la Evaluación de la Calidad del Agua (ICA), y lo propuesto por Fernández, et al. (2005) en el significado de interpretación de calidad para Norte de Santander, en su evaluación del rio Pamplonita, el agua del rio Tejo se cataloga según el ICA-NSF como Mediana (51-70) para cada uno de los tramos estudiados (Tabla 8). Esto confirma, la acelerada perturbación del agua de esta importante cuenca.
Así mismo, los parámetros fisicoquímicos analizados independientemente garantizan aguas de buena calidad. Por tanto, para el uso de este recurso hídrico, se debe reglamentar su uso según el Decreto 1594 del 84 para aguas destinadas para uso humano, recreativo y doméstico, lo que garantizaría a corto, mediano y largo plazo un mejor aprovechamiento del recurso del Rio Tejo para cualquier uso, pero con restricciones.

Tabla 8. Valores de Calidad del agua del rio Tejo, mediante el Índice ICA-NSF

\begin{tabular}{|c|c|c|c|c|c|}
\hline Parámetros & M1 & Estado & Ponderación & $q^{*} w$ & ICA \\
\hline Temperatura ${ }^{\circ} \mathrm{C}$ & 22 & 9 & 0,10 & 0,9 & \multirow{2}{*}{$\mathbf{M}$} \\
\hline Sólidos Disueltos mg/l & 60 & 85 & 0,08 & 6,8 & \\
\hline PH & 6,8 & 75 & 0,12 & 9 & $\mathbf{E}$ \\
\hline Oxígeno Disuelto mg/l & 80 & 88 & 0,17 & 14,96 & \multirow{2}{*}{$\mathbf{D}$} \\
\hline $\mathrm{DBO} 5 \mathrm{mg} / \mathrm{l} \mathrm{de} \mathrm{O} 2$ & $>30$ & 2.0 & 0,10 & 0,2 & \\
\hline Nitratos mg/l & 10 & 52 & 0,10 & 5,2 & I \\
\hline Fosfatos $\mathrm{mg} / \mathrm{l}$ & 0,25 & 93 & 0,10 & 9,3 & \multirow{4}{*}{$\mathbf{A}$} \\
\hline Turbidez UMT/ppm de SO4 & 20 & 62 & 0,08 & 4,96 & \\
\hline \multirow[t]{2}{*}{ Coliformes Fecales NMP/100 ml } & 300 & 33 & 0,15 & 4,95 & \\
\hline & & & 1,0 & 56,27 & \\
\hline Parámetros & M2 & Estado & Ponderación & $\mathrm{q}^{*} \mathrm{w}$ & ICA \\
\hline Temperatura ${ }^{\circ} \mathrm{C}$ & 23 & 9 & 0,10 & 0,9 & \multirow{2}{*}{$\mathbf{M}$} \\
\hline Sólidos Disueltos mg/l & 60 & 85 & 0,08 & 6,8 & \\
\hline $\mathrm{PH}$ & 6,5 & 70 & 0,12 & 8,4 & $\mathbf{E}$ \\
\hline
\end{tabular}



Cadena, Jose J; Gómez, Rubén D.

\begin{tabular}{|c|c|c|c|c|c|c|}
\hline Oxígeno Disuelto $\mathrm{mg} / \mathrm{l}$ & 75 & 72 & 0,17 & 12,24 & & \\
\hline $\mathrm{DBO} 5 \mathrm{mg} / \mathrm{l} \mathrm{de} \mathrm{O} 2$ & $>30$ & 2.0 & 0,10 & 0,2 & & \\
\hline Nitratos mg/l & 10 & 52 & 0,10 & 5,2 & & \\
\hline Fosfatos mg/l & 0,20 & 95 & 0,10 & 9,5 & & \\
\hline Turbidez UMT/ppm de SO4 & 20 & 62 & 0,08 & 4,96 & & \\
\hline \multirow[t]{2}{*}{ Coliformes Fecales NMP/100 ml } & 400 & 30 & 0,15 & 4,5 & & \\
\hline & & & 1,0 & 52,7 & & \\
\hline Parámetros & M3 & Estado & Ponderación & $\mathrm{q}^{*} \mathrm{w}$ & & ICA \\
\hline Temperatura ${ }^{\circ} \mathrm{C}$ & 23 & 9 & 0,10 & 0,9 & \multirow[b]{2}{*}{$\mathbf{M}$} & \\
\hline Sólidos Disueltos mg/l & 60 & 85 & 0,08 & 6,8 & & \\
\hline $\mathrm{PH}$ & 6,7 & 73 & 0,12 & 8,76 & \multirow[t]{2}{*}{$\mathbf{E}$} & \\
\hline Oxígeno Disuelto mg/l & 80 & 88 & 0,17 & 14,96 & & \\
\hline $\mathrm{DBO} 5 \mathrm{mg} / \mathrm{l} \mathrm{de} \mathrm{O} 2$ & $>30$ & 2.0 & 0,10 & 0,2 & D & \\
\hline Nitratos $\mathrm{mg} / \mathrm{l}$ & 10 & 52 & 0,10 & 5,2 & \multirow[t]{2}{*}{$\mathbf{I}$} & \\
\hline Fosfatos mg/l & 0,25 & 93 & 0,10 & 9,3 & & \\
\hline Turbidez UMT/ppm de SO4 & 20 & 62 & 0,08 & 4,96 & \multirow[t]{2}{*}{$\mathbf{A}$} & \\
\hline \multirow[t]{2}{*}{ Coliformes Fecales NMP/100 ml } & 300 & 33 & 0,15 & 4,95 & & \\
\hline & & & 1,0 & 56,03 & & \\
\hline
\end{tabular}


Fuente: Elaboración propia

\section{CONCLUSIONES}

El nivel de diversidad obtenido en esta investigación (1.71), se asume bajo por el poco tiempo en la realización de los muestreos, que sumados al resto de índices ecológicos como la riqueza (2.05), Dominancia (0.16), Equidad (0.83), permiten determinar la diversidad de la biota estudiada de una manera rápida y sencilla.

Numéricamente o estructuralmente la comunidad de macro invertebrados encontrados en este tramo de monitoreo posee diferencia en cada unidad, los cuales puede ser producto de los arrastres ocasionados en el cuerpo de agua, que implica las diferencias en cuanto a la abundancia de familias y cualitativamente a diferencias entre las familias. Sin embargo, cada taxón está bien representado, determinando sencillamente la calidad del agua en un lapso de tiempo relativamente corto.

Las variables biológicas medidas tanto con las condiciones fisicoquímicas interpretadas presentaron buenas condiciones ecológicas ambientales para toda el área de muestreo, considerando el agua del rio Tejo con una calidad aceptable, ligeramente contaminadas, en donde se evidencian efectos de contaminación moderada por materia orgánica.

\section{AGRADECIMIENTOS}

A la Universidad Francisco de Paula Santander, Facultad de Educación Artes y Humanidades, Grupo de Investigación GIFEAH, a la División de Investigación y Extensión (DIE) y muy especialmente a los
Jefes de Departamentos de Ciencias Básicas y Humanidades: Esp. Malka I. Cabellos M., y Esp. Claudia M. Dura.

\section{BIBLIOGRAFIA}

Abel, P (1989) Water pollution biology. Ellis Horwood, Chichester, United Kingdom. 387 pp.

Alba-Tercedor J. (1996). Macroinvertebrados acuáticos y calidad de las aguas de los Ríos, IV Simposio del Agua en Andalucía (SIAGA), Almeria, vol. 2, pp. 203-213.

Fernández, N.; Solano, F. (2005). Índices de calidad y de contaminación del agua., Universidad de Pamplona.

Hellawell, J.M. (1986). Biological indicators of freshwater pollution and environmental management. In, Pollution Monitoring Series, K. Mellanby (ed). Elsevier Applied Science Publishers, London, UK. 546pp.

Holdridge L. R. (1996). Ecologia basada en zonas de vida. Instituto Interamericano de Cooperación para la Agricultura (IICA). San José, Costa Rica.

NSF-National Sanitation Foundation. (2006). Consumer Information: Water Quality Index (WQI)., En http://www.nsf.org/consumer/just_for_ki ds/wqi.asp, Visitada: Noviembre de 2012.

Roldán G. (1996). Guía para el estudio de los macroinvertebrados acuáticos del departamento de Antioquia. Universidad de Antioquia. FEN Colciencias, Edit. Presencia, Bogotá. 234 pp.

Roldán, G. (2003). Bioindicación de la calidad del agua en Colombia. Uso del 
BMWP/Col, Ed. Universidad de Antioquia, Medellín. 182 p.

Rosenberg, D. M. and V. H. Resh. (1993). Freshwater biomonitoring and benthic macroinvertebrates. Chapman and Hall, New York.
Staub, R., J. W. Appling, A. M. Hofstetter \& J. Hass. (1970). The effects of industrial wastes of Memphis and Shelby County on primary planktonic producers. Biocscience Vol. 20: 905-912 pp.

Wihm J. F. \& T. C. Dorris. (1968). Biological parameters of water quality. Bioscience. Vol. 18: 447-481. pp. 